\title{
Trem2-mediated activation of microglia breaks link between amyloid and tau in Alzheimer's disease
}

John Hardy and Dervis Salih

Reta Lilla Weston Research Laboratories

Dementia Research Institute and Department of Neurodegenerative Disease, UCL Institute of Neurology

London WC1N 3BG

UK

\section{L.hardy@ucl.ac.uk}

The amyloid hypothesis of Alzheimer's disease is predicated on the data showing that APP mutations (and by implication $A \beta$ deposition) drives tangle formation in humans and also in doubly transgenic APP and MAPT mice $(1,2)$. It has been usually drawn as a cell autonomous process with $A ß$ outside neurons inducing tangle formation within the neuron. This cell autonomous view has become increasingly untenable (3). While genetic analysis of late onset Alzheimer's disease has implicated some loci involved in APP metabolism, the great majority have been shown to be involved in lipid metabolism and/or microglial metabolism (4) with, for example, TREM2 being a phospholipid receptor (5).

Identification of loss of function variants in TREM2 predisposing to AD, coincided with the demonstration that TREM2 expression increased with plaque load and that other microglial genes in this same expression module were also Alzheimer's risk genes (6-8). These data lead to the idea that genetic variability in response to amyloid deposition was the central predisposing commonality of many Alzheimer's risk genes (9).

While it has been previously proposed that disease-associated microglia may limit neuronal damage caused by amyloid pathology (10), the paper by Lee and colleagues (11), brings the role of these $A \beta$ activated microglia into sharper focus. They confirm that a mutant APP transgene increases tangle pathology in a mutant MAPT transgenic mouse, but then further show that this tangle formation is increased in the absence of TREM2. This observation suggests that 
an effective microglial response mitigates against amyloid induced tangle formation (Figure 1). In an important control experiment, they also show that the absence of TREM2 on its own does not increase tangle formation in MAPT mutant mice.

The simplest interpretation of these data is that amyloid induced membrane damage leads directly or indirectly to tangle formation and that microglia play an important role in resolving that damage. The fact that APP metabolism occurs largely at the synapse may explain why synaptic loss could be collateral damage in this process $(12,13)$. In the absence of an effective microglial damage response, amyloid induced damage continues unchecked and this then results in tangle formation and thus to neurodegeneration and dementia

1) Selkoe DJ, Hardy J. The amyloid hypothesis of Alzheimer's disease at 25 years. EMBO Mol Med. 2016 Jun 1;8(6):595-608.

2) Lewis J, Dickson DW, Lin WL, Chisholm L, Corral A, Jones G, Yen SH, Sahara N, Skipper L, Yager D, Eckman C, Hardy J, Hutton M, McGowan E. Enhanced neurofibrillary degeneration in transgenic mice expressing mutant tau and APP. Science. 2001 Aug 24;293(5534):1487-91. doi: 10.1126/science.1058189. PMID: 11520987.

3) De Strooper B, Karran E. The Cellular Phase of Alzheimer's Disease. Cell. 2016 Feb 11;164(4):603-15. doi: 10.1016/j.cell.2015.12.056. PMID: 26871627.

4) Jones L, Holmans PA, Hamshere ML, Harold D, Moskvina V, Ivanov D, Pocklington A, Abraham R, Hollingworth P, Sims R, Gerrish A, Pahwa JS, Jones N, Stretton A, Morgan AR, Lovestone S, Powell J, Proitsi P, Lupton MK, Brayne C, Rubinsztein DC, Gill M, Lawlor B, Lynch A, Morgan K, Brown KS, Passmore PA, Craig D, McGuinness B, Todd S, Holmes C, Mann D, Smith AD, Love S, Kehoe PG, Mead S, Fox N, Rossor M, Collinge J, Maier W, Jessen F, Schürmann B, Heun R, Kölsch H, van den Bussche H, Heuser I, Peters O, Kornhuber J, Wiltfang J, Dichgans M, Frölich L, Hampel H, Hüll M, Rujescu D, Goate AM, Kauwe JS, Cruchaga C, Nowotny P, Morris JC, Mayo K, Livingston G, Bass NJ, Gurling H, McQuillin A, Gwilliam R, Deloukas P, Al-Chalabi A, Shaw CE, Singleton AB, Guerreiro R, Mühleisen TW, Nöthen 
MM, Moebus S, Jöckel KH, Klopp N, Wichmann HE, Rüther E, Carrasquillo MM, Pankratz VS, Younkin SG, Hardy J, O'Donovan MC, Owen MJ, Williams J. Genetic evidence implicates the immune system and cholesterol metabolism in the aetiology of Alzheimer's disease. PLoS One. 2010 Nov 15;5(11):e13950. doi: 10.1371/journal.pone.0013950. Erratum in: PLoS One. 2011;6(2). doi:10.1371/annotation/a0bb886d-d345-4a20-a82eadce9b047798. Heun, Reinhard [added]; Kölsch, Heike [added]. PMID: 21085570 ; PMCID: PMC2981526.

5) Wang Y, Cella M, Mallinson K, Ulrich JD, Young KL, Robinette ML, Gilfillan S, Krishnan GM, Sudhakar S, Zinselmeyer BH, Holtzman DM, Cirrito JR, Colonna M. TREM2 lipid sensing sustains the microglial response in an Alzheimer's disease model. Cell. 2015 Mar 12;160(6):1061-71. doi: 10.1016/j.cell.2015.01.049. Epub 2015 Feb 26. PMID: 25728668; PMCID: PMC4477963.

6) Jonsson T, Stefansson H, Steinberg S, Jonsdottir I, Jonsson PV, Snaedal J, Bjornsson S, Huttenlocher J, Levey AI, Lah JJ, Rujescu D, Hampel H, Giegling I, Andreassen OA, Engedal K, Ulstein I, Djurovic S, IbrahimVerbaas C, Hofman A, Ikram MA, van Duijn CM, Thorsteinsdottir U, Kong A, Stefansson K. Variant of TREM2 associated with the risk of Alzheimer's disease. N Engl J Med. 2013 Jan 10;368(2):107-16. doi:

10.1056/NEJMoa1211103. Epub 2012 Nov 14. PMID: 23150908; PMCID: PMC3677583.

7) Guerreiro R, Wojtas A, Bras J, Carrasquillo M, Rogaeva E, Majounie E, Cruchaga C, Sassi C, Kauwe JS, Younkin S, Hazrati L, Collinge J, Pocock J, Lashley T, Williams J, Lambert JC, Amouyel P, Goate A, Rademakers R, Morgan K, Powell J, St George-Hyslop P, Singleton A, Hardy J; Alzheimer Genetic Analysis Group. TREM2 variants in Alzheimer's disease. N Engl J Med. 2013 Jan 10;368(2):117-27. doi: 10.1056/NEJMoa1211851. Epub 2012 Nov 14. PMID: 23150934; PMCID: PMC3631573.

8) Matarin M, Salih DA, Yasvoina M, Cummings DM, Guelfi S, Liu W, Nahaboo Solim MA, Moens TG, Paublete RM, Ali SS, Perona M, Desai R, Smith KJ, Latcham J, Fulleylove M, Richardson JC, Hardy J, Edwards FA. A genomewide gene-expression analysis and database in transgenic mice during 
development of amyloid or tau pathology. Cell Rep. 2015 Feb 3;10(4):633-44. doi: 10.1016/j.celrep.2014.12.041. Epub 2015 Jan 22. PMID: 25620700.

9) Salih DA, Bayram S, Guelfi S, Reynolds RH, Shoai M, Ryten M, Brenton JW, Zhang D, Matarin M, Botia JA, Shah R, Brookes KJ, Guetta-Baranes T, Morgan K, Bellou E, Cummings DM, Escott-Price V, Hardy J. Genetic variability in response to amyloid beta deposition influences Alzheimer's disease risk. Brain Commun. 2019;1(1):fcz022. doi:

10.1093/braincomms/fcz022. Epub 2019 Oct 10. PMID: 32274467; PMCID: PMC7145452.

10)Keren-Shaul H, Spinrad A, Weiner A, et al. A Unique Microglia Type Associated with Restricting Development of Alzheimer's Disease. Cell 2017; 169: 1276-1290.e17. PMID: 28602351.

11)Lee SH, Meilandt WJ, Xie L, Gandham VD, Ngu H, Barck KH, Rezzonico MG, Imperio J, Lalehzadeh G, Huntley MA, Stark KL, Foreman O, Carano RAD, Friedman BA, Sheng M, Easton A, Bohlen CJ, Hansen DV. Trem2 restrains the enhancement of tau accumulation and neurodegeneration by betaamyloid pathology. Neuron. 2021 Feb 27:S0896-6273(21)00084-2. PMID: 33675684.

12)Edwards FA. A Unifying Hypothesis for Alzheimer's Disease: From Plaques to Neurodegeneration. Trends Neurosci 2019; 42: 310-22. PMID: 31006494.

13)Bartels T, De Schepper S, Hong S. Microglia modulate neurodegeneration in Alzheimer's and Parkinson's diseases. Science. 2020; 370: 66-9. PMID: 33004513. 
Figure 1. Proposed interaction between TREM2 activation of microglia and $A \beta$ damage to neuronal membranes

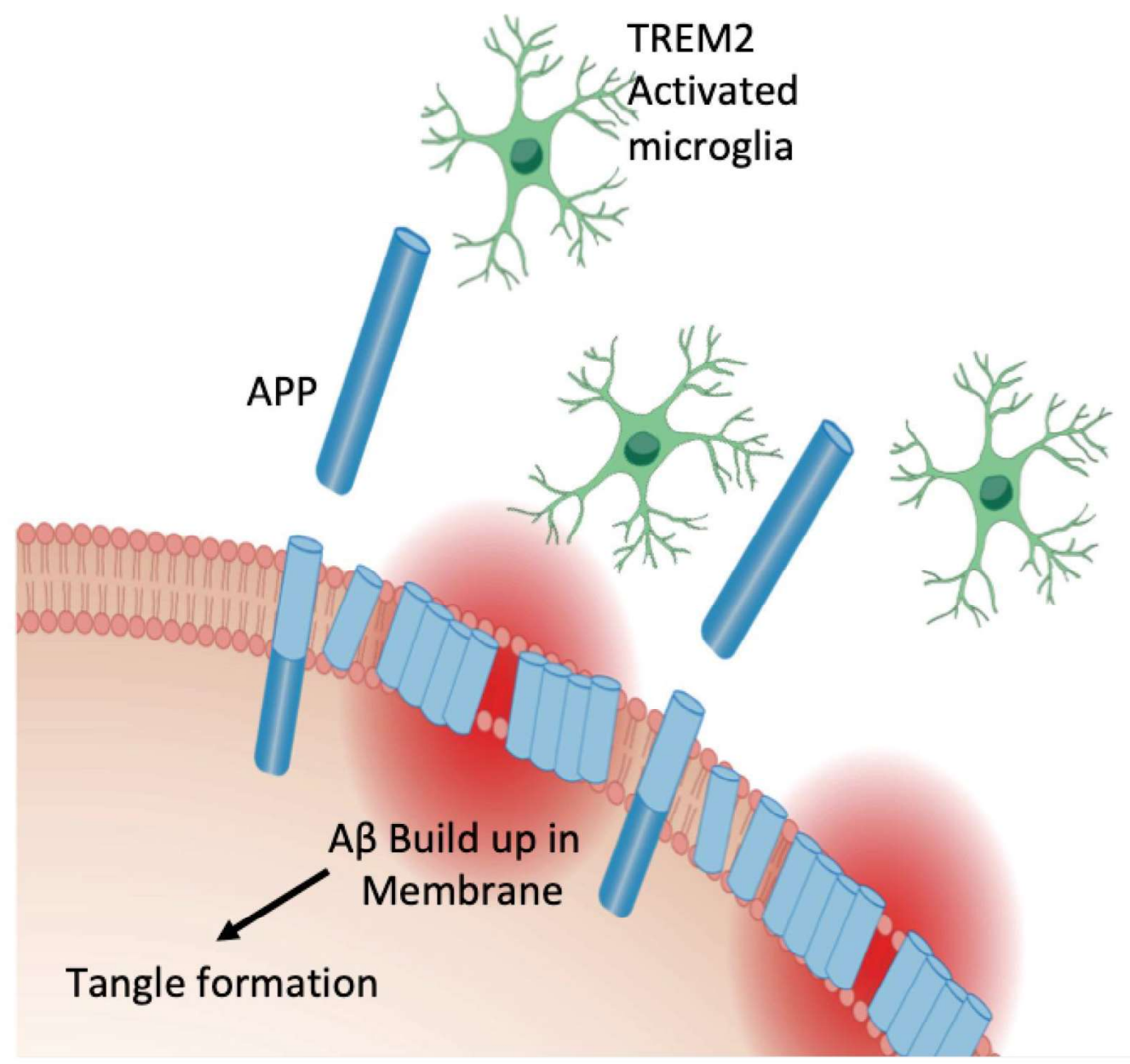

Suggested interpretation of the interaction between neuronal APP metabolism with $A \beta$ deposition disrupting membranes, attracting microglia, in part, through TREM2 signalling to repair or remove damaged membranes. When this process fails or is overwhelmed, tangle formation is instigated. 